\title{
Tinnitus and elderly: implications for quality of life
}

\begin{abstract}
Elderly has been occurring rapidly in the world. In recent years, there has been a significant increase in the number of individuals reaching the senescence stage, thus presenting several manifestations, among them, otoneurological complaints. These are commonly related to auditory and vestibular systems, the main ones being: hearing loss, dizziness, ear fullness and tinnitus. The association between elderly and otoneurology, several alterations can be observed, whether related to the biological process or to the environmental influence. Among the most common clinical symptoms, there is the complaint of tinnitus. The presence of tinnitus often becomes a factor of great negative repercussion in the individual's quality of life, bringing sleep difficult, problems concern concentration on daily and professional activities, issues in social life and, often, emotional disturbs, leading to states of anxiety and depression. ${ }^{1,2}$
\end{abstract}

Keywords: tinnitus, quality of life, elderly, environmental influence, otoneurology, hearing loss, dizziness, ear fullness, auditory, manifestations, emotional disturbs, anxiety, depression, social life, ears or head
Volume 2 Issue 4 - 2018

Lilian Felipe, Baylie Fox

Speech and Hearing Department, Lamar University, USA

Correspondence: Lilian Felipe, Speech and Hearing Department, Lamar University, USA, Email Ifelipe@lamar.edu

Received: July 20, 2018 | Published: August 09, 2018

\section{Introduction}

Tinnitus is a common complaint in the general population, and is defined as a sound in the head or ears that occurs in the absence of any external acoustic source. It can be classified in several ways, such as: characteristics (noise similar to the noise of rain, sea, running water, bells, insects, whistles, buzzing, bell, pulsation and others) (continuous or intermittent), intensity (tonal differences, intense or mild), in addition to being perceived in the ears or head. ${ }^{2,3}$ It is considered one of the three most important otoneurological manifestations, together with the complaints of hearing loss and dizziness, being often the main complaint, mainly in the elderly population. Thus, tinnitus is a symptom that frequently accompanies presbycusis (hearing loss related to the elderly process) and, according to the literature, is usually more disturbing than hearing loss itself. Usually, it is a disorder that produces extreme discomfort, being difficult to characterize and treat, and according to its severity can affect social interaction and even lead to suicide. Tinnitus is considered a physiological disorder resulting from abnormal neuronal activity in the auditory pathways and erroneously interpreted as sound in the cortical centers. Current concepts suggest that, in this condition, there is involvement of the peripheral and central, afferent and efferent auditory system and the interaction with other systems. The most current theories to explain its origin of this symptom defend the hypothesis that tinnitus occurs due to anomalous and spontaneous neuronal activity in the central auditory or non- auditory pathways, being a consequence of sensory deprivation after cochlear injury. ${ }^{4}$ Even though most cases are associated with a decrease in auditory threshold, tinnitus may be present in subjects with normal audiometry. ${ }^{5,6}$

Although there are several origins, such as otologic, neurological, infectious and drug changes, there is a strong association with exposure to noise and aging. The etiologies listed in the current scientific literature indicate that $35 \%$ of cases are of idiopathic origin, $18 \%$ are caused by sensorineural hearing loss, $15 \%$ presbycusis, ${ }^{7} 8.5 \%$ chronic exposure to noise, $4 \%$ acoustic trauma and $2 \%$ sudden deafness. The most frequent issues associated with tinnitus are: sleep disturbs, auditory complains, ${ }^{8}$ social problems and negative emotional reactions, such as anxiety and depression. Connections from the auditory system with the limbic system and the autonomic nervous system explain the development of tinnitus and the associated annoyances, similar to chronic pain. It is important to highlight that these comorbidities should be taken into account in assessing the impact of tinnitus on the individual's daily life. ${ }^{9}$ The literature presents as the most common cause or symptom associated with tinnitus is hearing loss. This relationship would be linear, that is, the greater the degree of hearing loss, the greater the nuisance. The presence of hearing loss would increase the risk of tinnitus provoking interference in concentration and emotional balance, or even that it functions as a cofactor of this interference. The classification of tinnitus requires a complete history and physical examination, complemented by appropriate diagnostic tests. Didactically, tinnitus may present as objective or subjective, according to the sound perception of the symptom..$^{10,11}$

The characteristics of the objective tinnitus are: pulsatile or click type, it is infrequent, can be heard by both patient and examiner, and is usually caused by sounds generated within the body, by vascular or muscular diseases. Regarding the intensity, it can be considered by degree as: light, when it is only perceived by the subject in certain situations; moderate, when the subject notices it, but does not bother; intense, when the unpleasant sensation brings disturbs, injuring and incapacitating the subject in various situations or activities. The description of the tinnitus characteristics by the individual is varied. It may sound like a "pure tone," a "whistle," a "buzz," a "whisper," and so on. It can be seen in one or both ears, or even in the head, without a specific side. It can be constant or intermittent, not being noticed for some periods. Its intensity can vary from light to very intense. Regarding the descriptive features of tinnitus, ${ }^{12} 52 \%$ reported the bilateral location, $10 \%$ in the head and less than $1 \%$ outside the head. There is no consensus on the unilateral localization in the affected ear. Because it is a symptom and can be found in several otologic and non -otological diseases, with a complex, multiple, and still not clear physiopathology, there are several therapeutic modalities that have been employed in the attempt of cure or even control of tinnitus. Some authors divide the treatment into two categories: those aimed at reducing the intensity of tinnitus and those aimed at relieving the discomfort associated with tinnitus. The first category includes 
pharmacotherapy and electrical stimulation and the other consists of pharmacotherapy, cognitive behavioral therapy, sound therapy, habituation therapy, massage and stretching, and hearing aids. There is no single treatment that is effective for all types of tinnitus. On the other hand, there are many treatments that decrease and even eliminate tinnitus.

\section{Quality of life}

The concept of "quality of life" refers to a movement intrinsic to the human and biological sciences, in order to value broad parameters, not only the control of symptoms, the reduction of mortality or the increase of life expectancy. Quality of life is an eminently human notion that has been approximated to the degree of satisfaction found in family, love, social and environmental life, ${ }^{13}$ and to existential aesthetics itself. The World Health Organization defines quality of life: "The individual's perception of their position in life within the context of their culture and the value system of where they live, and in relation to their goals, expectations, standards and concerns. It is a very broad concept that incorporates in a complex way the physical health of a person, their psychological state, their level of dependence, their social relations, their beliefs and their relation with prominent characteristics in the environment." Health-related with quality of life is defined as the value attributed to life balanced by functional deteriorations, by the perceptions and social conditions that are induced by diseases, injuries and treatments, and by the political and economic organization of the care system. In relation to the elderly group, quality of life includes the physical, psychological and social domains related to health, not only having the health situation, but also the elderly non-health aspects. The complaining of tinnitus often report sleep disturbances, difficulty concentrating, ${ }^{14}$ attention and symptoms of anxiety and/or depression. These complaints can cause difficulties at work, in the family and leisure environment, interfere with social habits and the dynamics of everyday life. Each patient is affected differently independent of presenting alterations in hearing levels, with great negative implications on quality of life and in the psychic state. Approximately $20 \%$ of patients with chronic tinnitus (when present for more than three months) present significant discomfort (and complaints such as: memory and concentration problems, speech discrimination and maintenance of selective attention, impairing leisure activities, rest, and social interaction, among others. ${ }^{15}$

\section{Elderly}

Population aging is one greatest triumph and also one of our greatest challenges. Global aging will increase social and economic demands around the world. However, older people are often ignored as a resource when in fact they are an important resource for the structure of our societies. Around the world, the proportion of people 60 and older is growing faster than any other age group. Between 1970 and 2025, the number of elderly people is expected to grow by $223 \%$, or around 694 million. By 2025 , there will be a total of approximately 1.2 billion people over the age of 60 . By 2050 there will be two billion, with $80 \%$ in developing countries. ${ }^{16}$ The aging of a population is associated with a reduction in the number of children and young people and an increase in the proportion of people aged 60 and over. As populations age, the triangular population pyramid of 2002 will be replaced by a more cylindrical structure by 2025 . Population aging is a natural process manifested by a decline in the functions of various organs. Characteristically, it tends to be linear in function of the time, not being able to define an exact point of transition, as in the other phases. It begins relatively early, at the end of the second decade of life, until the first functional and/or structural changes attributed to aging emerge at the end of the third decade. The natural process of aging involves innumerable biological transformations inherent in organisms that occur gradually and driven by evolutionary needs. The physiological changes intrinsic to aging are subtle, incapable of generating any incapacity in the initial phase, although, over the years, they will cause increasing levels of limitations to the performance of basic activities of daily living. ${ }^{17}$

Elderly is a natural and inevitable process. It is a phase that is part of the biological and natural life cycle, a social and historical construction due to the varied ways in which the aging process is understood and lived in different communities. Aging is a heterogeneous process, under the influence of socio-cultural, political and economic aspects, in a dynamic and permanent interaction with the biological and subjective dimension of individuals, a current challenge, affecting countries developed or not. Active aging is an ideology that applies to both individuals and population groups, enabling people to realize their potential, participate in society according to their specifics, and provide them with adequate protection, security and care when needed. Its goal is to increase healthy life expectancy by ensuring quality of life; including for individuals who are frail, physically inactive or in need have care. Tinnitus is a factor of intense dissatisfaction in the elderly patient, as it impairs their daily activities in addition to providing emotional and sleeps alterations, correlating the presence of arterial hypertension in patients with tinnitus although they have not found a correlation between the audiometric degree of hearing loss and the level of discomfort felt by the subject. Tinnitus is a multifaceted and complex symptom that can dramatically compromise the individual's quality of life. ${ }^{18,19}$

\section{Final considerations}

It is important to consider the need for better planning of health interventions in the elderly population, in addition to the training of health professionals in relation to aging and tinnitus, leading them to early interventions, which would prevent the cascade effect of functional decline and impairment quality of life.

\section{Acknowledgements}

None.

\section{Conflict of interest}

The author declares there is no conflict of interest.

\section{References}

1. Baguley D, McFerran D, Hall D. Tinnitus. Lancet. 2013;382(9904):16001607.

2. Bhatt JM, Lin HW, Bhattacharyya N. Prevalence, Severity, Exposures, and Treatment Patterns of Tinnitus in the United States. JAMA Otolaryngol Head Neck Surg. 2016;142(10):959-965.

3. Cima RFF, Crombez G, Vlaeyen JWS. Catastrophizing and Fear of Tinnitus Predict Quality of Life in Patients With Chronic Tinnitus. Ear Hearing. 2011;32(5):634-641.

4. Durai M, O'Keeffe MG, Searchfield GD. The Personality Profile of Tinnitus Sufferers and a Nontinnitus Control Group. J Am Acad Audiol. 2017;28(4):271-282. 
5. Fioretti AB, Fusetti M, Eibenstein A. Association between sleep disorders, hyperacusis and tinnitus: Evaluation with tinnitus questionnaires. Noise Health. 2013;15(63):91-95.

6. Global Health and Aging. World Health Organization, 2011.

7. Han BI, Lee WH, Kim YT, et al. Tinnitus: characteristics, causes, mechanisms, and treatments. J Clin Neurol. 2009;5(1):11-19.

8. Heller AJ. Classification and epidemiology of tinnitus. Otolaryngol Clin North Am. 2003;36(2):239-48.

9. Hennig TR, Costa MJ, Urnau D. Recognition of speech of normal-hearing individuals with tinnitus and hyperacusis. Int Arch Otorhinolaryngol. 2011;15:21-28.

10. Henry JA, McMillan GP, Thielman EJ, et al. Evaluating psychoacoustic measures for establishing presence of tinnitus. J Rehabil Res Dev. 2013;50(4):573-584.

11. Jastreboff PJ. Phantom auditory perception (tinnitus): mechanisms of generation and perception. Neurosci Res. 1990;8(4):221-254.
12. Kaltenbach JA. The dorsal cochlear nucleus as a participant in the auditory, attentional and emotional components of tinnitus. Hear Res. 2006;216-217:224-234.

13. Lewis JE, Stephens SD, Mckenna L. Tinnitus and Suicide. Clin Otolaryngol Allied Sci. 1994;19(1):50-54.

14. Nondahl DM, Cruickshanks KJ, Wiley TL. The 10-year incidence of tinnitus among older adults. Int J Audiol. 2010;49(3):580-585.

15. Pinto PCL, Sanchez TG, Tomita S. The impact of gender, age and hearing loss on tinnitus severity. Braz J Otorhinolaryngol. 2010;76(1):18-24.

16. Teixeira AR, Rosito LP, Gonçalves AK, et al. Tinnitus in Elderly Individuals: Discomfort and Impact in the Quality of Life. Int Arch Otorhinolaryngol. 2017;21(1):66-71.

17. Tyler R, Coelho $\mathrm{C}$, Tao $\mathrm{P}$, et al. Identifying tinnitus subgroups with cluster analysis. Am J Audiol. 2008;17(2):S176-S184.

18. Ukaegbe OC, Orji FT, Ezeanolue BC, et al. Tinnitus and Its Effect on the Quality of Life of Sufferers: A Nigerian Cohort Study. Otolaryngol Head Neck Surg. 2017;157(4):690-695. 